\title{
Prenatal Detection of Critical Congenital Heart Disease
}

\author{
${ }^{1}$ Lina W Irshaid, ${ }^{2}$ Najwa Elfky, ${ }^{3}$ Badreldeen Ahmed
}

\begin{abstract}
Congenital heart disease (CHD) is a leading cause of infant mortality and $30 \%$ fetuses born with CHDs have other associated malformations and chromosomal abnormalities. Prenatal diagnosis also allows parents to opt for termination of the pregnancy.
\end{abstract}

Keywords: Critical congenital heart disease, Fetal echocardiography, Prenatal diagnosis.

How to cite this article: Irshaid LW, Elfky N, Ahmed B. Prenatal Detection of Critical Congenital Heart Disease. Donald School J Ultrasound Obstet Gynecol 2016;10(2):131-135.

Source of support: Nil

Conflict of interest: None

\section{INTRODUCTION}

When discussing congenital heart disease (CHD), consideration of certain points is critical. The first consideration is that CHD is a leading cause of infant mortality, with an estimated incidence of about 4 to 13 per 1000 live births. ${ }^{1,2}$ Moreover, between 1950 and 1994, 42\% of infant deaths reported to the World Health Organization were attributable to cardiac defects. ${ }^{3}$ The second consideration is that $30 \%$ of fetuses born with CHDs have other associated malformations or chromosomal abnormalities. The third consideration is that the vast majority of CHDs affect pregnancies with no previously known risk factors. The fourth consideration is that prenatal diagnosis of CHDs improves the outcome in certain cardiac conditions, which include hypoplastic left heart, transposition of the great arteries, and coarctation of the aorta. ${ }^{4,5}$ In special situations, prenatal diagnosis of CHDs gives parents the choice of the place of delivery. Prenatal diagnosis may also allow parents the option of termination of the pregnancy.

Antenatal diagnosis of cardiac defects did not witness a great improvement over the last few years. ${ }^{6,7}$ Furthermore, prenatal detection rates of CHD vary

\footnotetext{
1-3Medical Student

${ }^{1}$ Weill Cornell Medical School, Qatar

${ }^{2}$ Weill Cornell Medicine, Al Luqta St, Ar-Rayyan, Qatar

${ }^{3}$ Feto maternal Centre, Doha, Qatar

Corresponding Author: Badreldeen Ahmed, Feto maternal Centre, Weill Cornell Medical School, Qatar, Phone: 0097455845583, e-mail: profbadreldeen@hotmail.com
}

widely. ${ }^{8}$ Some of this variation can be attributed to differences in examiner experience, maternal obesity, transducer frequency, abdominal scars, gestational age at the time of ultrasound examination, amniotic fluid volume, and fetal position. ${ }^{9,10}$ Because of the above, efforts are being made to try to improve the detection rates and diagnosis of CHD. These efforts include classroom courses, online courses, training in referred centers, and introduction of guidelines and protocols to diagnose $\mathrm{CHD}$ by professional bodies, such as the International Society of Ultrasound in Obstetrics and Gynecology (ISUOG).

\section{HOW TO EXAMINE THE FETAL HEART DURING ROUTINE ANOMALY SCAN}

A strict protocol should be followed, which should include the subsequent points. Firstly, examination of all fetuses should be done at gestational age between 18 and 22 weeks. Secondly, the abdominal probe should be done using high-resolution ultrasound. The zoom and cine loop facilities should be used when needed. Thirdly, in all fetuses, a four-chamber view of the heart, with comments on situs, atrial chambers, ventricular chambers, and atrioventricular junction and valves (Figs 1 and 2) should be viewed. The left and right ventricular outflow tracts should be examined. Fourthly, outflow tracts are imaged by tilting the probe toward the fetal head. The great arteries should be of equal size and should cross at approximately $90^{\circ}$ (Figs 3 and 4). Fifthly, all fetuses should have a three-vessel view, which enabled diagnosis of conditions such as coarctation of the aorta, right aortic arch, double aortic arch, and vascular rings (Fig. 5). Sixthly, all fetuses had a three-vessel and trachea view. Using this view, the transverse aortic arch and its relationship to the trachea could be demonstrated. Seventhly, color Doppler mapping should be used to examine the various cardiac structures and to improve the detection of abnormal blood flow. Many congenital heart defects can be seen on the standard four-chamber view (Table 1). However, four-chamber views are not enough to diagnose all cardiac malformations because of many factors, such as the anomaly could be far away from the four-chamber view, the anomaly evolved in utero, routine checklist may be overlooked, and inadequate examination.

Examination of the outflow and outflow trachea tract is essential to diagnose other congenital cardiac defects (Table 2). 


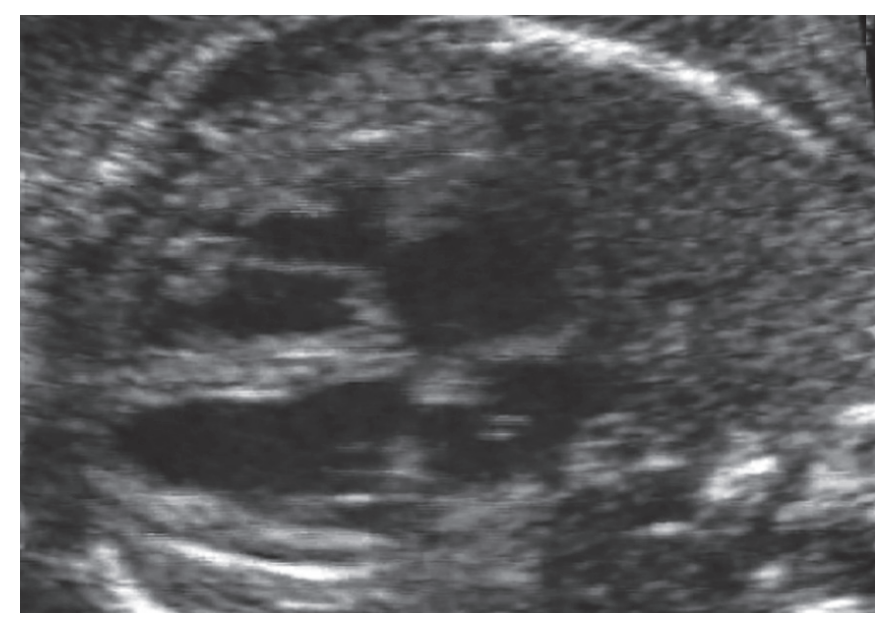

Fig. 1: Four-chamber view, showing both atria of similar size, ventricles of similar size, foramen ovale flap in the left atrium

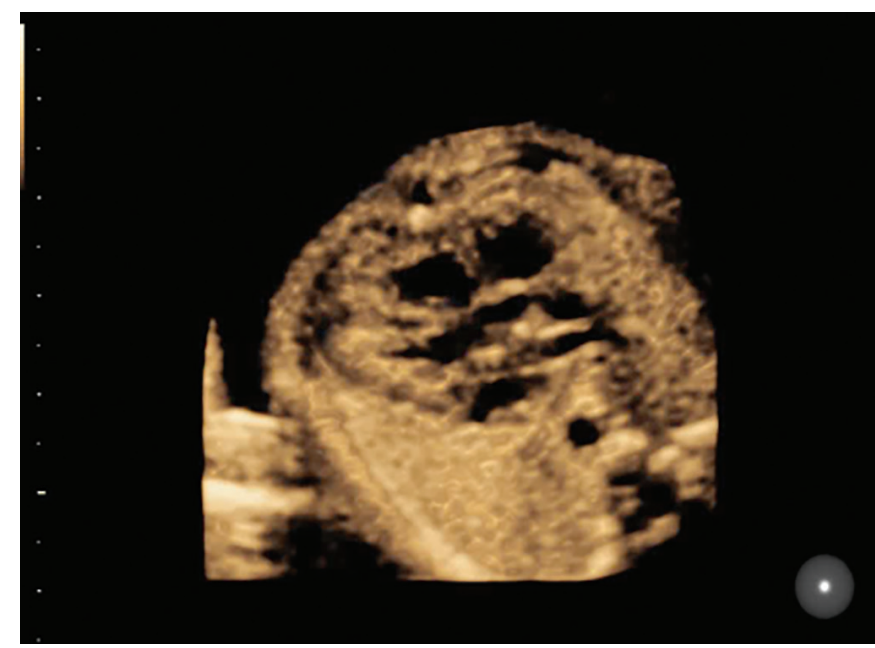

Fig. 3: Left outflow tract. The anterior aortic wall is continuous with the intact interventricular septum

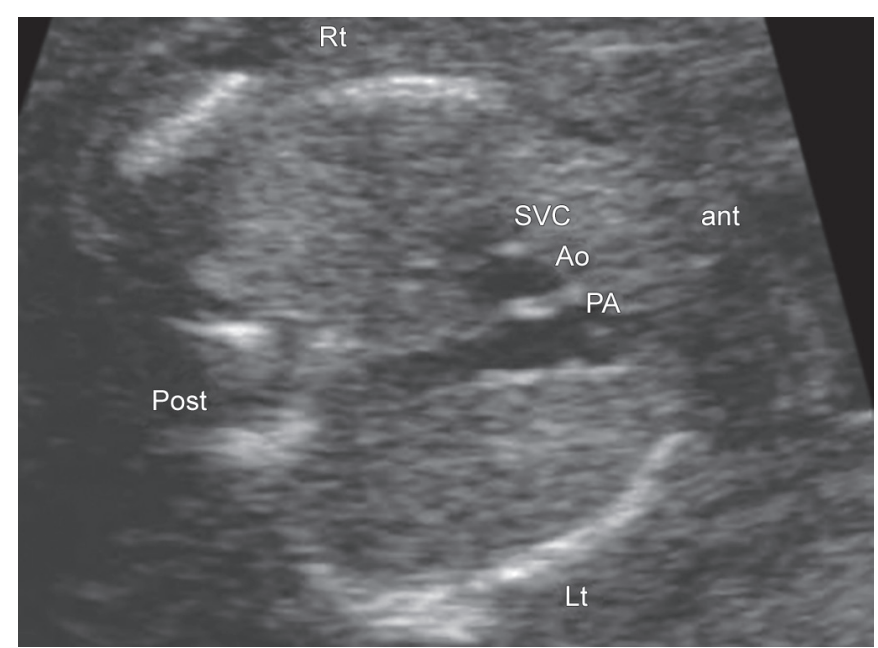

Fig. 5: The three-vessel view. This view demonstrates the relationship between superior vena cava, aorta, and pulmonary artery. Note the correct position and the size of the three vessels as well as correct alignment

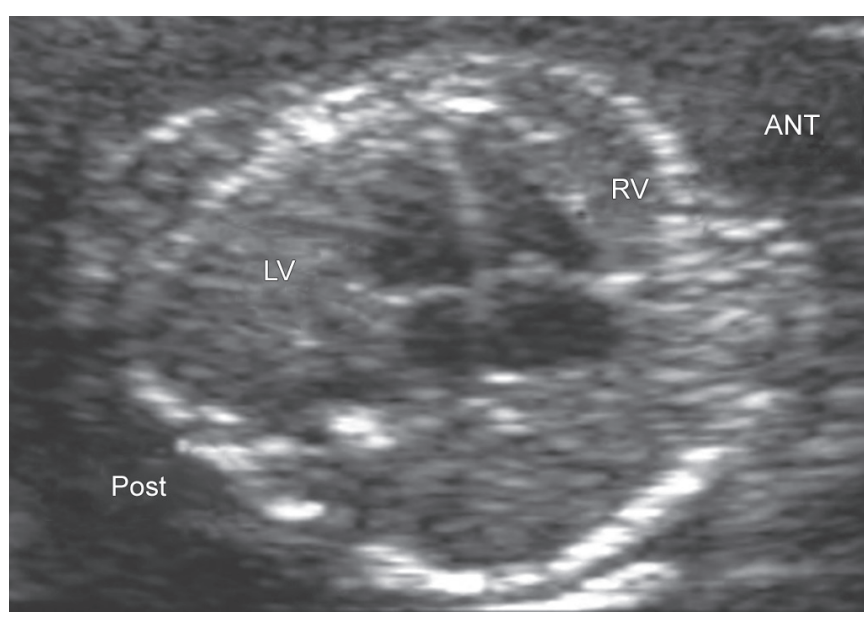

Fig. 2: Four-chamber view showing both ventricles on equal size intact septum, moderator band seen in the right ventricle, and different levels of attachment of atrioventricular valve

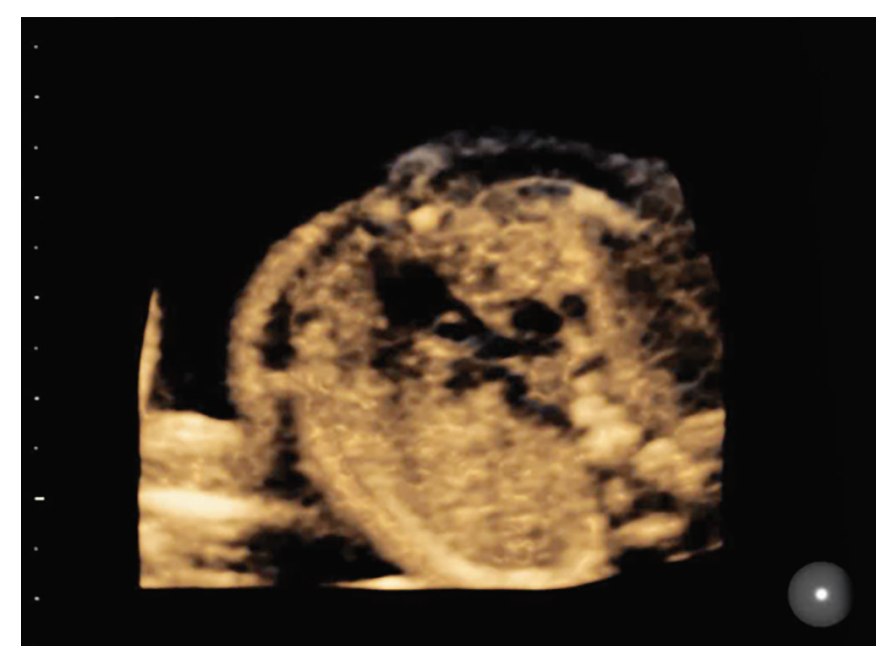

Fig. 4: Right outflow tract. The pulmonary artery bifurcates immediately. The pulmonary valve moves freely

Table 1: Congenital cardiac defects detected on four-chamber view

- Hypoplastic right and left ventricle

- Atrioventricular canal defect

- Double inlet ventricle

- Ebstein's anomaly

- Single ventricle

- Cardiac tumors

- Large ventricular septal defect

Table 2: Congenital cardiac defects missed on four-chamber view

- Transposition of the great vessels

- Tetralogy of Fallot

- Double outlet right ventricle

- Truncus arteriosus

- Small/moderate ventricular septal defect

- Atrial septal defect

- Coarctation of the aorta

- Total anomalous pulmonary venous return

- Aortic stenosis

- Pulmonary stenosis 


\section{DISCUSSION}

When we examine the fetal heart during anomaly scan, we aim to screen for any critical congenital heart defect which may affect the life of the newborn. Detection and diagnosis are distinct terms. Detection is the process of discovering a finding, while diagnosis is the process of identifying a disease by examination. In many fetomaternal centers, such as our center in Doha, detection rate is very high, as we were able to discover 10 out of 11 cases of CHD. The accuracy of our diagnosis of CHD is $100 \%$, as all those who were referred to the pediatric cardiologist had their diagnosis confirmed postnatally via echocardiography. This result demonstrates that a high detection rate and accurate diagnosis of CHD is possible.

Such result is very encouraging as only a few years ago the detection rate of CHD was suboptimal. A European study between 2000 and 2005 involving 16 countries and including $3.3 \times 10^{6}$ live births showed the prenatal detection rate of CHD is only $20 \%$. Many other studies done in European countries showed that the detection rate varies dramatically, between 20 and $80 \%$, emphasizing the need for additional studies and an improvement in detection rate. ${ }^{11-13}$

Another study by Galindo et $\mathrm{al}^{14}$ involving 33 centers in Spain, and including 1,060 major CHDs between 2004 and 2006, reported that the prenatal detection rate of CHD in routine second-trimester scans was as low as $52.6 \%$ (95\% confidence interval (CI) 45.6-60.8). The study identified two important predictors of an increased detection rate by center: firstly, a systematic approach to the examination of the heart, showing at least the fourchamber view and the outflow tracts (prevalence ratio 1.3, 95\% CI 1.0-1.8) and secondly, the availability of specialists in fetal echocardiography to perform the examination (prevalence ratio 1.4, 95\% CI 1.1-1.9). ${ }^{15}$ The study advised that prenatal detection of CHD should be globally strengthened by way of a uniform approach to the cardiac examination along with improving examiner training and skills. Moreover, authors of the study recommended close collaboration with fetal heart specialists to help accomplish this aim. ${ }^{15}$ A prospective observational study at a London teaching hospital reported similar conclusions. Authors encouraged continuous feedback-based training of health care professionals, a low threshold for echocardiography referrals, and convenient access to fetal heart specialists as techniques to improve the effectiveness of a screening for CHDs. ${ }^{11}$

A study by Marek et al, ${ }^{16}$ including all pediatric and fetal patients with CHD over a 21-year period in the Czech Republic, reported a discrepancy in the detection rate of various cardiac defects. For example, the prenatal

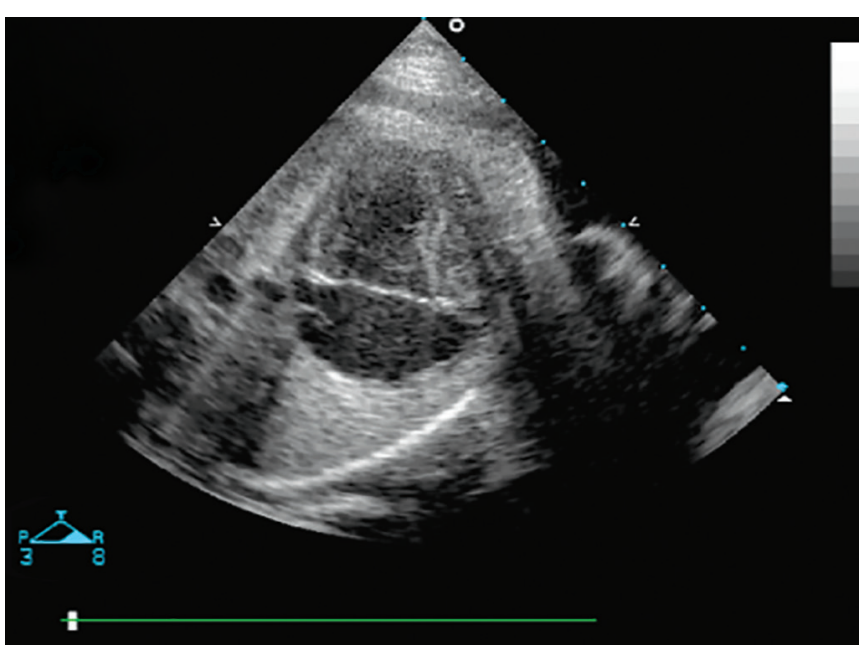

Fig. 6: Hypoplastic heart. This condition may be present at the time of anomaly scan at 20 weeks or may evolve as the result of atrioventricular valve stenosis

detection rate for hypoplastic left heart was $95.8 \%$ (Fig. 6), whereas detection of transposition of the great arteries was only $25.6 \%$.

Prenatal detection of CHD is essential because it has a large impact on the appropriate management of affected infants during labor and delivery. For example, some babies may need to be delivered in a cardiac operation room for quick intervention. When the fetus is delivered, there are critical changes in the fetal circulation which include initiation of breathing, conversion of the fetal circulation from a parallel circuit to a series circuit, increase in the systemic vascular resistance, decrease in pulmonary vascular resistance, and closure of fetal shunt pathways. Detection of CHD during the antenatal period allows parents and physicians to choose the place of delivery and prepare a multidisciplinary team for the baby's arrival, which includes a fetal medicine specialist, pediatric cardiologist, neonatologist, anesthesiologist, and pediatric cardiac surgeon. These preparations improve the prognosis of the infant.

The accepted gestational age for performing fetal echocardiography is between 18 and 22 weeks. However, a small proportion of CHDs become evident or more obvious as pregnancy progresses. Thus, the cardiac evaluation can be normal at 18 weeks, although a significant malformation is diagnosed later on in the pregnancy or after birth. This is true of some cases of aortic or pulmonary stenosis, cardiac tumors, and cardiomyopathies (Fig. 7). Fortunately, it is rare for a life-threatening malformation to arise after 20 weeks' gestation. In addition, minor lesions, such as small ventricular septal defects can be overlooked because of the limitation of ultrasound resolution (Fig. 8). Other defects, such as persistent arterial duct and atrial septal defect cannot be predicted prenatally as these communications are always present 


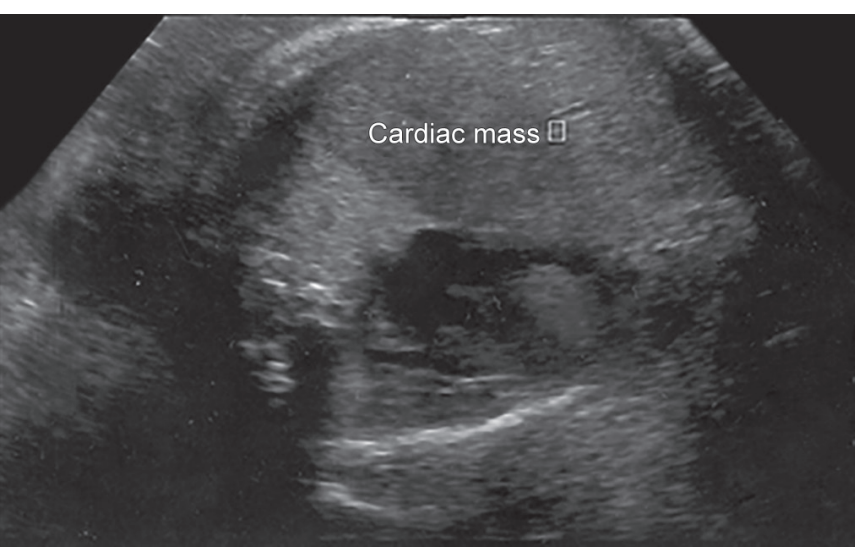

Fig. 7: A case of rhabdomyoma which is a benign cardiac tumor; however, it could be associated with tuberous sclerosis which is autosomal dominant condition

prenatally. Thus, there are confidence limits with even detailed fetal heart scanning. These confidence limits may be much wider with poor image quality. For this reason, it is crucial to counsel the parents on these limitations of antenatal scanning and to ensure that they are aware that while prenatal screening presents a huge benefit to their fetus, the detection is not $100 \%$.

\section{WHAT IS NEW?}

\section{The New 3D/4D-based Spatiotemporal Imaging Correlation in Fetal Echocardiography}

Spatiotemporal imaging correlation is an automated device incorporated into the ultrasound probe and has the capacity to perform slow sweep to acquire a single three-dimensional (3D) volume. This acquired volume is composed of a great number of two-dimension (2D) frames. ${ }^{17,18}$ This volume can be analyzed and reanalyzed as required to demonstrate all the required cardiac views. It also provides the examiner with the ability to review all images in a looped cine sequence. Spatiotemporal imaging correlation has the potential to improve our ability to diagnose CHD. This potential becomes possible because of unlimited number of images available for viewing, additional information not available in the standard 2D ultrasound images, information can be shared with other colleagues remote from the site of examination for second opinion, and the patient can be counseled in great detail in light of new information.

In conclusion, the majority of prenatally detectable cases of $\mathrm{CHD}$ occur in patients without any risk factors or extracardiac anomalies. Several studies have reported the detection rate of $\mathrm{CHD}$; however, these figures vary widely. Regardless, these studies all came down to the general agreement that an improvement in detection rate is necessary, as it can greatly improve the prognosis of the

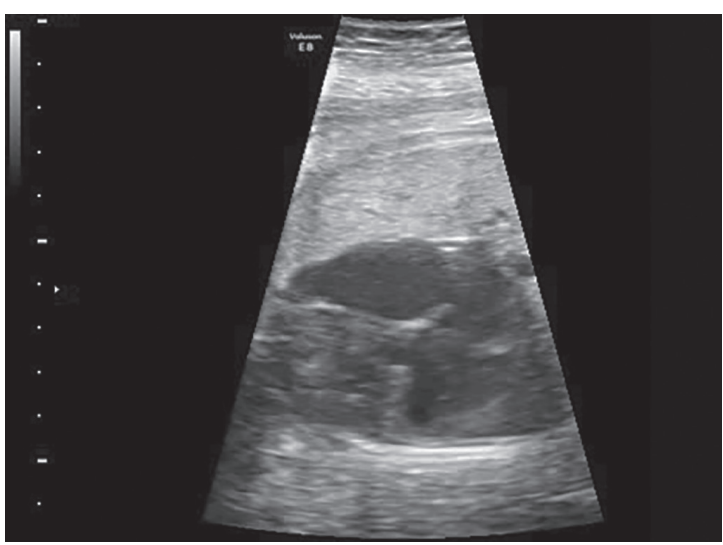

Fig. 8: Ventricular septal defect can occur in any part of the interventricular septum and is often associated with other cardiac defects

infant. It also allows hospital systems to prepare for the arrival of the infant by making sure a multidisciplinary team is available. Detection rate can be improved by implementing a uniform, systematic approach to the fetal cardiac examination and by ensuring that fetal echocardiography is performed by specialists who are familiar with the prenatal diagnosis of CHD.

\section{REFERENCES}

1. Ferencz C, Rubin JD, McCarter RJ, Brenner JI, Neill CA, Perry LW, Hepner SI, Downing JW. Congenital heart disease: prevalence at livebirth. The Baltimore-Washington Infant Study. Am J Epidemiol 1985 Jan;121(1):31-36.

2. Cuneo BF, Curran LF, Davis N, Elrad H. Trends in prenatal diagnosis of critical cardiac defects in an integrated obstetric and pediatric cardiac imaging center. J Perinatol 2004 Nov; 24(11):674-678

3. Rosano A, Botto LD, Botting B, Mastroiacovo P. Infant mortality and congenital anomalies from 1950 to 1994: an international perspective. J Epidemiol Community Health 2000 Sep;54(9):660-666.

4. Crane JP, Lefevre ML, Winborn RC, Evans JK, Ewigman BG, Bain RP, Frigoletto FD, McNellis D. A randomized trial of prenatal ultrasonographic screening: impact on the detection, management, and outcome of anomalous fetuses. The RADIUS Study Group. Am J Obstet Gynecol 1994 Aug;171(2):392-399.

5. Abu-Harb M, Hey E, Wren C. Death in infancy from unrecognized congenital heart disease. Arch Dis Child 1994 Jul;71(1):3-7.

6. Bonnet D, Coltri A, Butera G, Fermont L, Le Bidois J, Kachaner J, Sidi D. Detection of transposition of the great arteries in Fetuses reduces neonatal morbidity and mortality. Circulation 1999 Feb 23;99(7):916-918.

7. Tworetzky W, Wilkins-Haug L, Jennings RW, Van Der Velde ME, Marshall AC, Marx GR, Colan SD, Benson CB, Lock JE, Perry SB. Balloon dilation of severe aortic stenosis in the fetus: potential for prevention of hypoplastic left heart syndrome: candidate selection, technique, and results of successful intervention. Circulation 2004 Oct 12;110(15):2125-2131.

8. Simpson LL. Screening for congenital heart disease. Obstet Gynecol Clin North Am 2004 Mar;31(1):51-59. 
9. Devore GR, Medearis AL, Bear MB, Horenstein J, Platt LD. Fetal echocardiography: factors that influence imaging of the fetal heart during the second trimester of pregnancy. J Ultrasound Med 1993 Nov;12(11):659-663.

10. Sharland GK, Allan LD. Screening for congenital heart disease prenatally. Results of a $21 / 2$-year study in the South East Thames region. Br J Obstet Gynaecol 1992 Mar;99(3):220-225.

11. Carvalho JS, Mavrides E, Shinebourne EA, Campbell S, Thilaganathan B. Improving the effectiveness of routine prenatal screening for major congenital heart defects. Heart 2002 Oct;88(4):387-391.

12. Yagel S, Weissman A, Rotstein Z, Manor M, Hegesh J, Anteby E, Lipitz S, Achiron R. Congenital heart defects. Natural course and in utero development. Circulation 1997 Jul 15;96(2): 550-555.

13. Stumpflen I, Stumpflen A, Wimmer M, Bernaschek G. Effect of detailed fetal echocardiography as part of routine prenatal ultrasonographic screening on detection of congenital heart disease. Lancet 1996 Sep 28;348(9031):854-857.
14. Galindo A, Herraiz I, Escribano D, Lora D, Melchor JC, de la Cruz J. Prenatal detection of congenital heart defects: a survey on clinical practice in Spain. Fetal Diagn Ther 2011;29(4):287-295.

15. Dolk H, Loane M, Garne E; European Surveillance of Congenital Anomalies Working Group. Congenital heart defects in Europe: prevalence and perinatal mortality, 2000 to 2005. Circulation 2011 Mar 1;123(8):841-849.

16. Marek J, Tomek V, Skovránek J, Povysilová V, Samánek M. Prenatal ultrasound screening of congenital heart disease in an unselected national population: a 21-year experience. Heart 2011 Jan;97(2):124-130.

17. DeVore GR, Falkensammer P, Sklansky MS, Platt LD. Spatiotemporal image correlation: new technology for evaluation of the fetal heart. Ultrasound Obstet Gynecol 2003 Oct;22(4): 380-387.

18. DeVore GR, Polanko B. Tomographic ultrasound imaging of the fetal heart: a new technique for identifying normal and abnormal cardiac anatomy. J Ultrasound Med 2005 Dec;24(12):1685-1696. 